\title{
Analysis of Prospect of Application Laser induced bending forming technology in the field of aerospace
}

\author{
Cailing $\mathrm{Li}^{1, \mathrm{a}^{*}}$, Chao Cui ${ }^{1, \mathrm{~b}}$, Xiaoyu Shen ${ }^{1, \mathrm{c}}$, Bo Wang ${ }^{1}$,Zhe Wang ${ }^{1}$, Xiaoming \\ Lai $^{1}$, Xin Liu ${ }^{1}$, ShuFen Liu ${ }^{1}$, Jiaowen Liu ${ }^{1}$ \\ ${ }^{1}$ Beijing spacecrafts, Beijing,China \\ a lindali6@126.com, ${ }^{\mathrm{b}}$ cuichao20012001@163.com, ${ }^{\mathrm{c}}$ xiaoyu.shen@163.com
}

Key words: Laser induced; flexible forming; spacecraft; application prospect

Abstract. Laser induced forming is a kind of dieless forming technology, which is caused by the uneven internal thermal stress caused by local transient heating. In this paper, the characteristics of the laser flexible thermal forming technology are introduced, and the application prospect of the laser induced forming technology is analyzed. It lays the foundation for the popularization and application of laser induced forming technology in the field of aerospace.

\section{Introductions}

Laser induced forming technology applied to sheet metal was developed in the mid-1980s, and it was firstly proposed by Japanese scholars. The forming mechanism is shown in Fig. 1. It is a new flexible and dieless forming technology, based on thermal expansion and contraction and thermal expansion characteristics of the internal organization of the material. Laser scans the metal sheet surface by a certain track, and the thermal stress generates in the area of the heated region, which distributions are non-uniform. Plastic deformation of sheet metal occurs under thermal stress, in order to achieve sheet metal bending, shaping, or forming a three-dimensional complex surface.

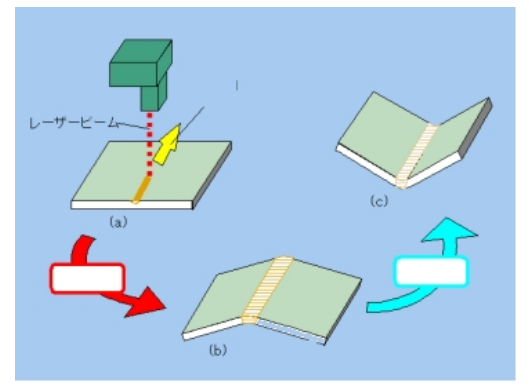

Fig.1 a schematic view of the mechanism [1]

Sheet metal forming occupies an important proportion in the manufacture field of the spacecrafts. In order to meet the increasingly development requirements of diverse spacecraft structures, how to take full advantage of the performance of sheet metal forming, how to develop new forming process, how to use computer technology to optimize the parts structure and process, and how to detect and control the forming process, are inevitable trends of sheet metal forming technology. Therefore, the laser induced forming technology, as a high-precision, good 
controllability, flexible and dieless forming technology in the field of aerospace, has great application prospect.

\section{Characteristics of laser induced forming technology}

Compared with the conventional forming process, laser induced forming technology has the following characteristics [2-6].

1 Laser induced forming technology is a flexible and dieless forming technology. Dieless forming greatly shortens the development cycle and reduces the costs. Flexible forming especially suites for the development of diverse structures, single small-batch or large-lot production.

2 Laser induced forming technology is a non-contact forming technology. In the process, the force does not act directly on the part. Springback does not occur, thus forming precision is better.

3 Laser induced forming technology is a heat accumulation process. The area of single laser scanning and the amount of deformation are very small. The total deformation amount is formed by a plurality of scans. And deformation is always generated in the hot state. Difficult-to-deformable materials at room temperature can also be bent, such as titanium.

4 In theory, by optimizing process parameters and scanning traces, any bent part can be available through laser induced forming technology, such as cylindrical, conical, spherical and other complex three-dimensional surface parts.

5 Laser induced forming technology enables closed-loop control on the whole process of CNC laser machine, by using infrared thermometer and shape measuring instrument. It can fix parts online to ensure the quality and forming precision of the part.

6 Since the laser beam has a good direction and coherence, so that the laser induced forming technology can be applied to the deformation of the structures whose space is restricted.

7 The laser induced forming technology can achieve a variety of processes at the same station, such as bending, cutting, welding, etc.

While the research on laser induced forming technology is still at the starting stage, because of its unique advantages, people have felt its great potential benefits. Laser forming induced technology in the fields of aerospace, automotive, shipbuilding microelectronics and others will have broad application prospect.

\section{Application prospect analysis of laser induced forming technology in the field of aerospace}

A large number of light alloy plate structures are applied to the spacecraft components, especially in the manned spacecraft. Most structures are made of light alloy plate by bending. These structures can be divided into the following four categories.

(1) Bulkheads and stringers, used to enhance the strength and stiffness of the cabin seal or seal 
structures of the spacecraft.

(2) Box-shaped products and binding belts, used for brackets of cabin equipment

(3)Large complex surface, including spherical, cylindrical, conical, and other complex shape frame-covering and light weight panels, used for multi-surfaces of sealed cabin structures and the stiffeners and shields of unsealed structures.

Taking into account the development requirements of the above three types of light alloy structures of the spacecraft, in view of the characteristics of laser induced forming technology; it has been applied to the spacecraft. This technology will have great prospect, if it can be applied to the forming of bulkheads, stringers, box-shaped products, binding belts, frame-coverings and light weight panels.

Laser induced forming technology for the binding belt. The binding belt is an important part, which is applied to the unlocking device between the satellite and the rocket. Its role is to connect the satellite and the clamps of the mating frame of the rocket to ensure a reliable connection. Difficult-to-deformable materials at room temperature are selected, whose deformation needs to be achieved by springback dies, shaped dies, repeated reconditioning, long development cycle and high cost. By adjusting the process parameters, laser induced forming can quickly accomplish precision forming of the binding belt. The shaping effect is shown in Fig.2 Laser induced forming technology application prospect in the field of aerospace is revealed.

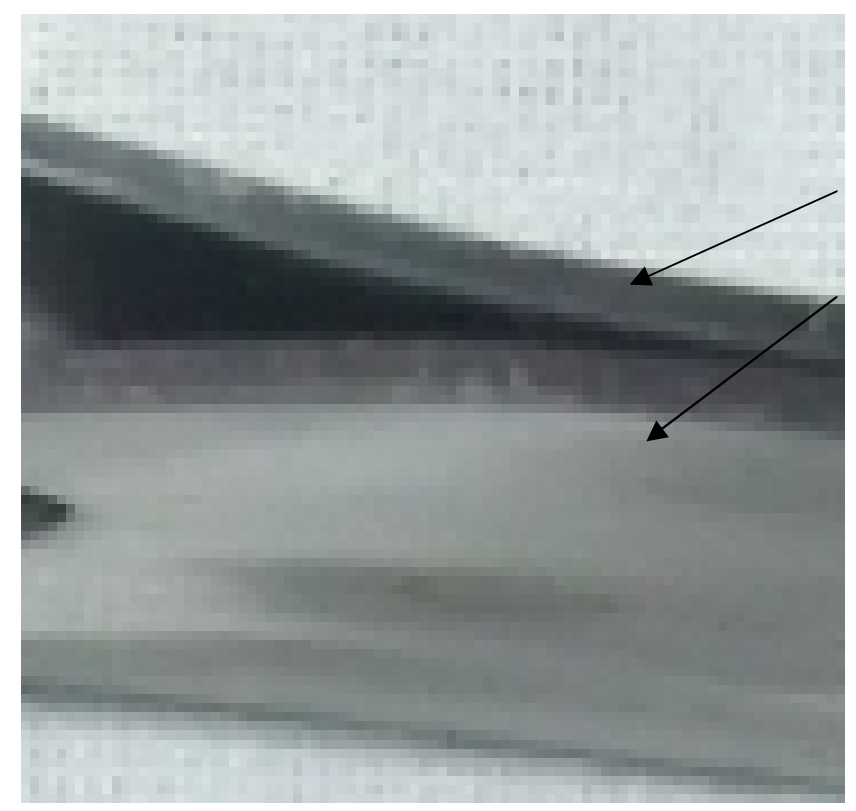

Laser induced forming area

Fig. 2 The effect schematic of the binding belt

The laser induced forming technology for the light weight panel .The light weight panel is the most important structure of the spacecraft sealed compartment. The light weight panel is made from a single piece of sheet in the machining process. It has the following characteristics: flexible geometries, various forms of the tendons, variable frame-covering thicknesses. It is easy to easy to achieve structural weight reduction and equal strength design. It can improve sealing reliability and 
anti-fatigue performance, and greatly enhance the strength and stiffness of the structures. Thus, light weight panels will be the most important structures of domestic and foreign spacecraft sealed compartments for a long period of time. Fig.3 shows the International Space Station and the new generation of light weight panels of foreign spacecraft.
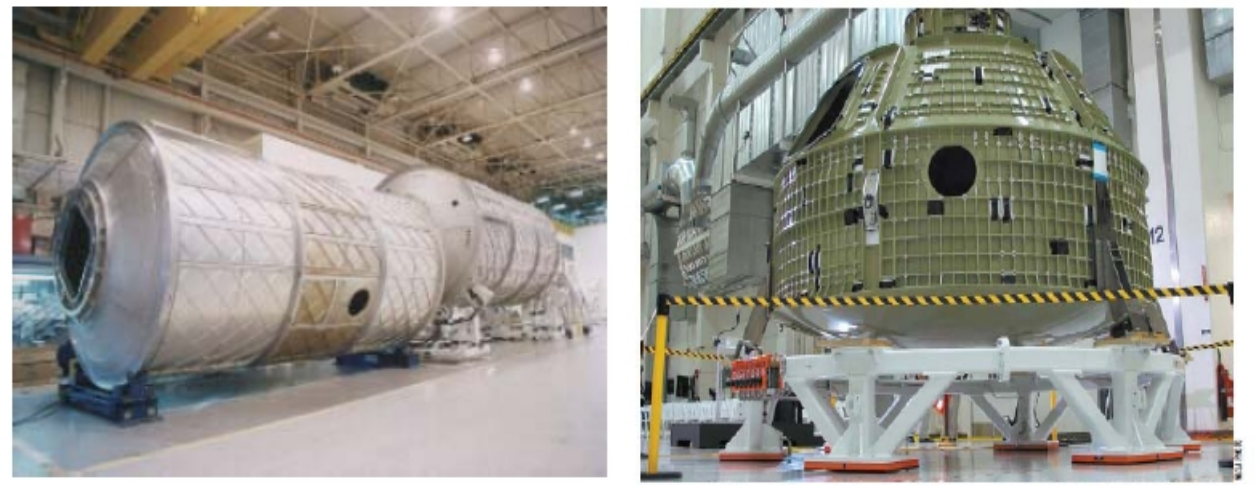

Fig.3 The International Space Station and the new generation of light weight panels of foreign spacecraft[7]

Although light weight panel structure has a series of advantages, but due to the integrated design, the difficulty of its formation has increased dramatically. With the start of the space station project in China, lightweight panels will present characteristics of various forms and high-precision. At the same time, high-precision forming technology will become more urgent.

Taking into account these features of the laser induced forming technology, the column section of the light weight panel is formed by using laser induced bending (Fig.4). The material of the light weight panel is aluminum. The central angle is $45^{\circ}$. It reveals that laser induced forming technology has broad application prospect in the field of aerospace.

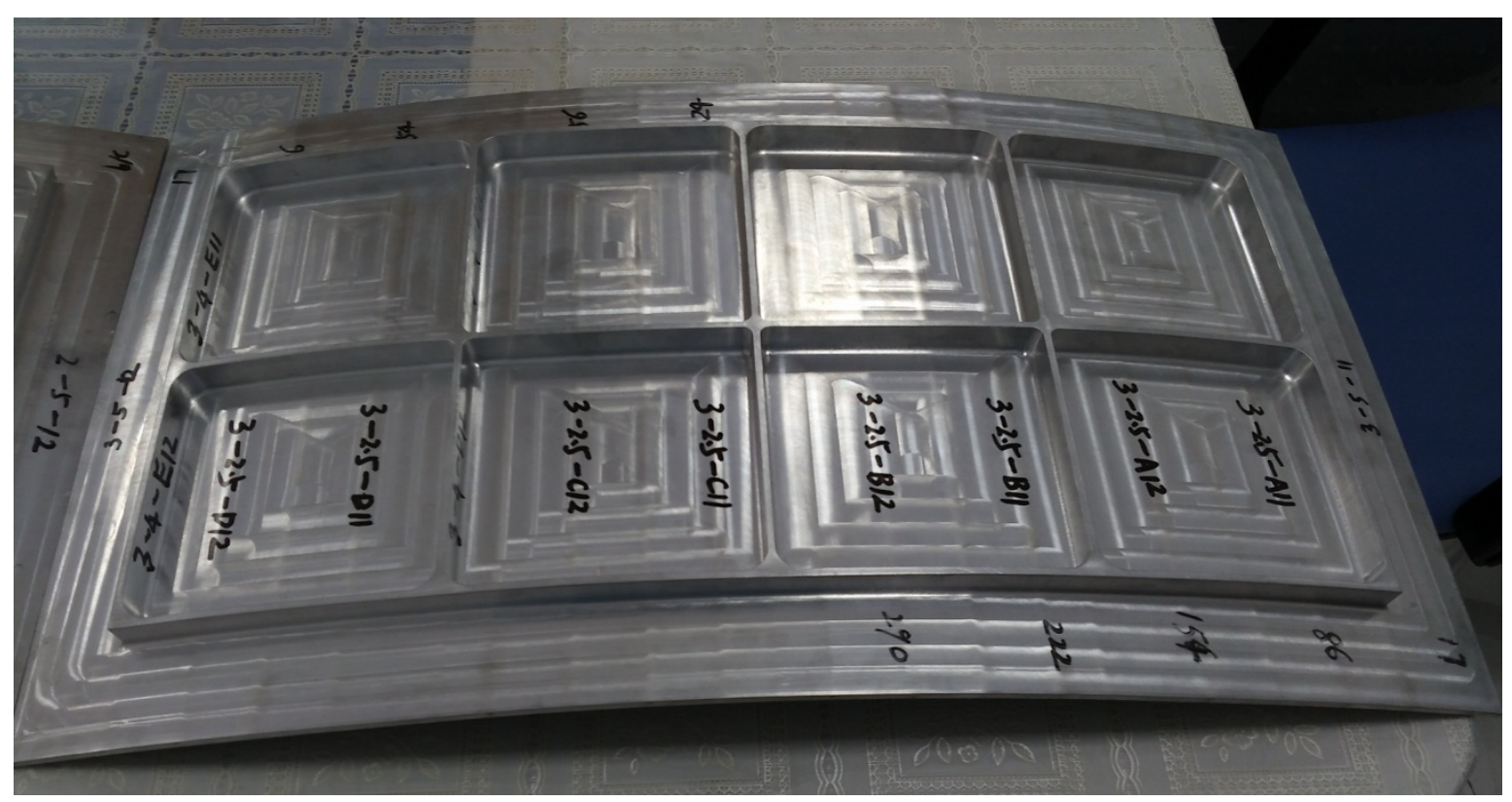

Fig.4 The light weight panel 


\section{Conclusions}

The laser induced forming technology solves the problem of the binding belt forming. It implements a flexible high-precision forming of the light weight panel. Therefore it will have broad application prospect in the field of aerospace, which is new and applicable to sheet metal bending.

\section{References}

[1] Nameba Y. Laser forming in space[A]. Wang C P.Proc.of Int.Conf On Lasers85ed[C]. 1986.403-407

[2] Geiger M. Synergy of laser material processing and metal forming[J]. Annals of the CIRP, 1994,43 2 :563-570.

[3] Wenchuan Li and Lawrence Yao Y. Laser Forming with Constant Line Energy[J]. The International Journal of Advanced Manufacturing Technology, 2001,17 3 :196-203.

[4] Cheng P J, Lin S C. An analytical model for the temperature field in the laser forming of sheet metal[J]. Journal of Materials Processing Technology, 2000,101:260 267.

[5] Zhong Ji Shi Wu Research applied to the sheet material deformation field of laser forming [J] Plastic Engineering. 199852 33-38

[6] Hennige T, Holzer S, Vollertsen F, et al. On the working accuracy of laser bending [J]. Journal of Materials Processing Technology, 1997, 71:422-432.

[7]Damage Tolerance Behavior of Friction Stir Welds in Aluminum, NASA report. 